\title{
Anti-glial fibrillary acidic protein antibodies as a cause of reversible splenial lesion syndrome (RESLES): a case report
}

\author{
Kundian Guo ${ }^{1} \cdot$ Xiaohui Lai $^{1} \cdot$ Yue Liu ${ }^{1} \cdot$ Dong Zhou ${ }^{1} \cdot$ Zhen Hong $^{1,2} \circledast$
}

Received: 9 April 2021 / Accepted: 1 June 2021 / Published online: 5 June 2021

(c) Fondazione Società Italiana di Neurologia 2021

\section{Introduction}

Reversible splenial lesion syndrome (RESLES) is a spectrum of disorders radiologically characterized by reversible lesions mainly involving the splenium of the corpus callosum [1]. RESLES most commonly occurs in patients with seizures, particularly following antiepileptic drugs withdrawal. RESLES can also cause by infections, highaltitude cerebral edema, hypoglycemia, hyper/hyponatremia, cerebral venous thrombosis, several non-epileptic pharmacological agents, and miscellaneous conditions including anorexia nervosa, malnutrition, vitamin B12 deficiency, Charco-Marie-Tooth disease, systemic lupus erythematosus, and eclampsia [1-3]. Autoimmune encephalitis as a rare condition associated with RESLES was reported in only one anti-NMDAR encephalitis, one anti-VGKC encephalitis, and one anti-Yo encephalitis case, respectively [3-5].

Autoimmune glial fibrillary acidic protein (GFAP) astrocytopathy is a novel immunotherapy-responsive autoimmune inflammatory central nervous system (CNS) disorder

Kundian Guo and Xiaohui Lai have contributed equally to the manuscript.

\section{Zhen Hong}

hongzhengoog@aliyun.com

Kundian Guo

93502563@qq.com

Xiaohui Lai

441607140@qq.com

Yue Liu

979642317@qq.com

Dong Zhou

zhoudong66@yahoo.de

1 Department of Neurology, West China Hospital, Sichuan University, No.37 Guoxue Road, Chengdu 610041, Sichuan, China

2 Department of Neurology, Chengdu Shangjin Nanfu Hospital, Chengdu, Sichuan, China characterized by anti-GFAP antibodies positive encephalitis, meningoencephalitis, or meningoencephalomyelitis [6]. Brain MRI abnormalities are commonly observed. Lesions involve the subcortical white matter, basal ganglia, hypothalamus, brainstem, cerebellum, meninges, and ventricle [6]. Approximately $50 \%$ of patients demonstrated a radial linear periventricular enhancement pattern, considered as a radiological hallmark of autoimmune GFAP astrocytopathy [6].

Herein, we report a rare case of an adult confirmed with autoimmune GFAP astrocytopathy presenting brain MRI imaging that indicates RESLES. To the best of our knowledge, this is the first case to provide direct evidence for correlations between anti-GFAP antibodies and RESLES.

\section{Case report}

A previously healthy 23 -year-old male presented with a 5-day history of headache and psychosis. Neurologic examination revealed decreased cognition and consciousness level, stiff neck, and weakness (grade 4/5) in both lower extremities. The deep tendon reflexes of his upper and lower extremities were increased. The Babinski sign was present bilaterally and meningeal signs were positive.

CSF studies revealed lymphocyte-predominant pleocytosis of $80 / \mu \mathrm{L}$ white blood cells ( $90 \%$ lymphocyte, normal range: $0-5 / \mu \mathrm{L})$ and elevated protein $(1.24 \mathrm{~g} / \mathrm{L}$, normal range: $0.15-0.45 \mathrm{~g} / \mathrm{L}$ ), with remarkable elevated opening pressure of $285 \mathrm{~cm} \mathrm{H}_{2} \mathrm{O}$. Microbiological exams in serum and CSF including serological tests, PCR methods, and culture were negative (Table 1). No infectious agent was found in CSF using metagenomics next-generation sequencing. GFAP antibodies (CSF, 1:10) were detected within patient CSF using indirect immunofluorescence (cell-based assay) on HEK293 cells (Euroimmun, Lübeck, Germany) (Fig. 1A). There were no other detectable onconeuronal or neuronal cell surface antibodies, anti-AQP4 antibodies, or anti-MOG antibodies in CSF or serum. Oligoclonal band test in serum 
Table 1 Additional auxiliary examination results
Cerebrospinal fluid

WBC

Glucose

80/ $\mu \mathrm{L}$ (90\% lymphocyte)

Protein

Microbiological exams

Bacteria (culture)

(-)

$1.24 \mathrm{~g} / \mathrm{L}$

Mycobacterium tuberculosis (culture)

$(-)$

Cryptococcus (culture)

$(-)$

Herpes simplex virus 1 and 2 (PCR)

$(-)$

$(-)$

Cytomegalovirus (PCR)

Epstein-Barr virus (PCR)

Japanese encephalitis virus (PCR)

$(-)$

$(-)$

$(-)$

Metagenomics next-generation sequencing (-)

${ }^{a}$ Onconeuronal antibodies

(CSF/serum)

Anti-Hu

$(-/-)$

Anti-Yo

$(-/-)$

Anti-Ri

$(-/-)$

Anti-CV2

$(-/-)$

Anti-amphiphysin

$(-/-)$

Anti-Ma2

$(-/-)$

Anti-SOX1

$(-/-)$

Anti-Tr

$(-/-)$

Anti-GAD65

$(-/-)$

Anti-Zic4

$(-/-)$

Anti-titin

$(-/-)$

Anti-recoverin

$(-/-)$

${ }^{a}$ Neuronal cell surface antibodies

(CSF/serum)

Anti-N-methyl-D-aspartate receptor

$(-/-)$

Anti-alpha-amino-3-hydroxy-5-methyl-4-isoxazolepropionic acid receptor 1

Anti-alpha-amino-3-hydroxy-5-methyl-4-isoxazolepropionic acid receptor 2

Anti-gamma-aminobutyric acid type A receptor

$(-/-)$

$(-/-)$

$(-/-)$

$(-/-)$

$(-/-)$

$(-/-)$

Anti-contactin-2-associated protein receptor

$(1: 10 /-)$

$(-/-)$

$(-/-)$

$(-/-)$

Oligoclonal band (CSF/serum)

Hematologic tests

Routine blood test

$(-)$

Biochemical test

C-reactive protein

Microbiological tests

Human immunodeficiency virus

$(-)$

TORCH

Epstein-Barr virus

Parasites

$(-)$

Mycobacterium tuberculosis

(-)

Tumor markers

Carcino-embryonic antigen

$(-)$

Cancer antigen 15-3
(-) 
Table 1 (continued)

\begin{tabular}{ll}
\hline Cancer antigen 19-9 & $(-)$ \\
Cancer antigen 125 & $(-)$ \\
Cancer antigen 72-4 & $(-)$ \\
Total prostate-specific antigen & $(-)$ \\
Free prostate specific antigen & $(-)$ \\
Neuron-specific enolase & $(-)$ \\
Cytokeratin 19 fragment antigen 21-1 & $(-)$ \\
Immunological test & \\
IgG4 & $(-)$ \\
Rheumatoid factor & $(-)$ \\
AKA & $(-)$ \\
ANA & $(-)$ \\
Anti-dsDNA & $(-)$ \\
c-ANCA-IgG & $(-)$ \\
p-ANCA-IgG & $(-)$ \\
MPO-ANCA-IgG & $(-)$ \\
PR3-ANCA-IgG & $(-)$ \\
Anti-SS-A & $(-)$ \\
Anti-SS-B & $(-)$ \\
Anti-Scl-70 & $(-)$ \\
Anti-Jo-1 & $(-)$ \\
Anti-RNP & $(-)$ \\
Anti-ACA & $(-)$ \\
Thyroid antibodies & \\
Thyroid globulin antibodies & $(-)$ \\
Thyroid peroxidase antibodies & $(-)$ \\
Thyroid stimulating hormone receptor antibodies & $(-)$ \\
\hline
\end{tabular}

${ }^{\mathrm{a}}$ Cell-based assay (Euroimmun, Lübeck, Germany)

$W B C$, white blood cell; $C S F$, cerebrospinal fluid; $P C R$, polymerase chain reaction; TORCH, toxoplasma, treponema pallidum, rubella virus, cytomegalovirus, and herpes simplex virus 1 and $2 ; A K A$, anti-keratin antibodies; $A N A$, anti-nuclear antibody; $d s D N A$, double-stranded deoxyribonucleic acid; $A N C A$, anti-neutrophil cytoplasmic antibodies; anti-SM, anti-Smith antibodies; $R N P$, ribonuclear protein; $A C A$, anticardiolipin

and CSF were negative. Other hematologic test results including routine blood test, biochemical test, vitamin B12 level, C-reactive protein, microbiological test, tumor markers, immunological test, and thyroid profiles were unremarkable (Table 1). Electroencephalogram exhibited diffuse slow activities. Initial brain magnetic resonance imaging (MRI) revealed a single lesion in the splenium of the corpus callosum (SCC) hypointense on T1-weighted, hyperintense on T2-weighted, and FLAIR sequences (Fig. 1C-E). Brain MRA and spinal MRI were negative.

Although 5 days of intravenous corticosteroid pulses (intravenous methylprednisolone $1000 \mathrm{mg} /$ day) and IVIg ( $0.4 \mathrm{~g} / \mathrm{kg} / \mathrm{day})$ were administered, the illness still progressed rapidly for the further 14 days. The patient developed coma, seizures, blurred vision, and hypoventilation and was intubated for acute respiratory failure. Following transfer to the neurological intensive care unit and treatment with 5-day additional intravenous corticosteroid pulses (intravenous methylprednisolone $1000 \mathrm{mg} /$ day) and IVIg ( $0.4 \mathrm{~g} / \mathrm{kg} /$ day $)$ therapy, the patient gradually regained consciousness and strength after 2 weeks and was finally extubated on day 50 after onset. Repeat brain MRI exhibited complete resolution of the SCC lesion (Fig. 1F-H).

Anti-GFAP antibodies were negative both in sera and CSF after 4 months. The patient made a full recovery after a 10-month follow-up. Autoimmune GFAP astrocytopathy (anti-GFAP meningoencephalitis) and RESLES were the presumed diagnoses. 
Fig. 1 Auxiliary examinations of the case with autoimmune GFAP astrocytopathy and RESLES. Antibody test in serum and CSF through indirect immunofluorescence on HEK293 cells (Euroimmun, Lübeck, Germany): A Patient CSF demonstrated binding to the surface of cells expressing GFAP proteins $(1: 10)$. B Regions with no specific fluorescence to other proteins on the same slide, analyzed simultaneously, were regarded as negative controls (scale bar: $75 \mu \mathrm{m}$ ). Brain MRI: C-E Initial brain MRI on admission (5 days after onset) exhibited an isolated splenium of the corpus callosum (SCC) lesion (arrows) with hypointensity on T1WI, hyperintensity on T2WI, and on FLAIR. F-H On day 50 after onset (10 days after the second course of immunotherapy), repeat MRI highlighted complete resolution of the SCC lesion
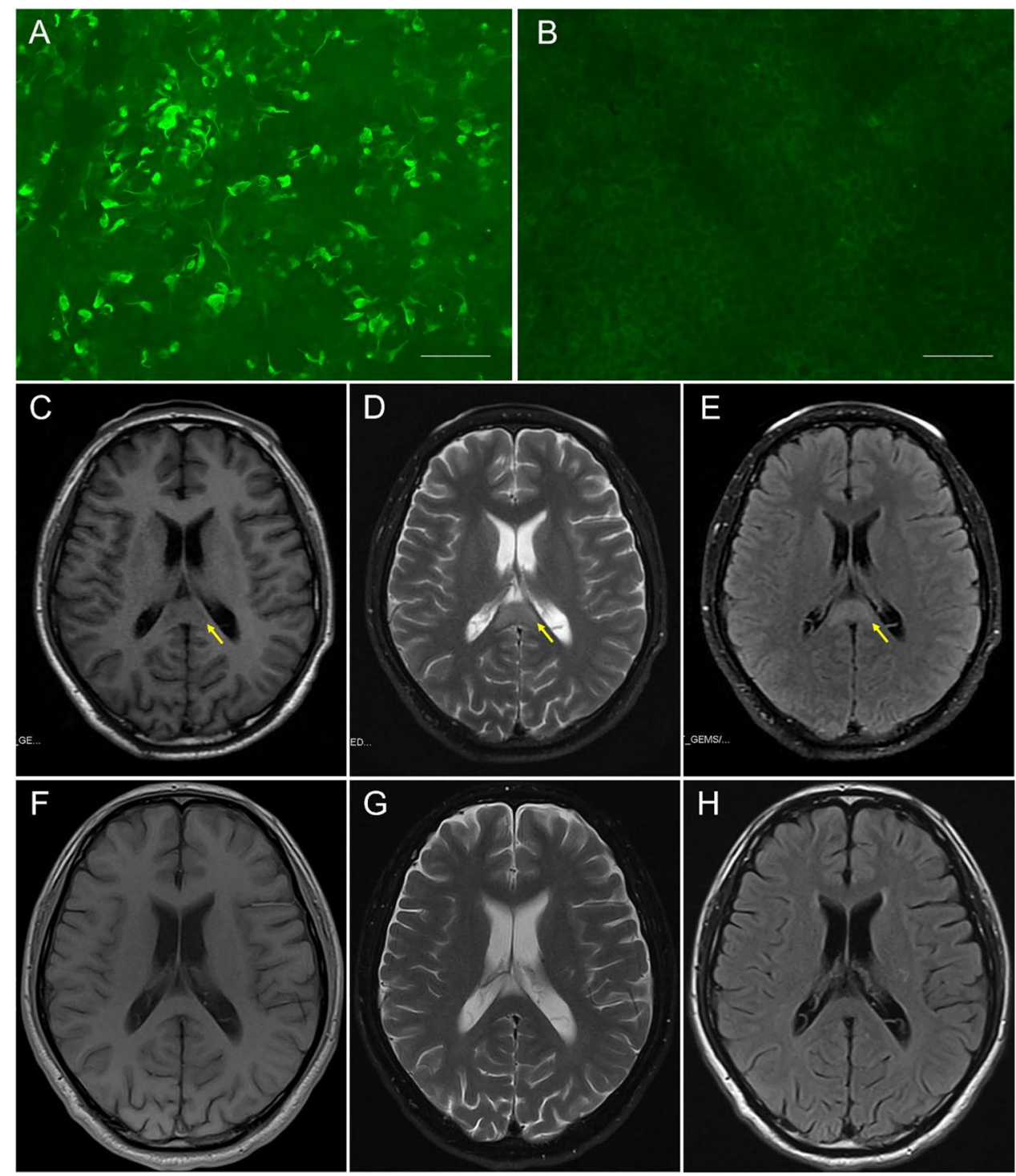

\section{Discussion}

Other known causes of RESLES were extensively excluded during diagnosis in this case. Seizures and pharmacological agents were considered as possible etiologies for RESLES. However, no patient history of seizures was present, with the SCC lesion being identified prior to seizure development and consequent treatment. Viral or other pathogenic infections seemed unlikely since no fever or other sign of infection was present, with all microbiological exams including metagenomics next-generation CSF sequencing were negative. Besides, the subsequent complete reversion of the lesion makes ischemic causes seem impossible. Moreover, biochemical, vitamin B12 level, rheumatologic, and thyroid-antibody tests exhibited normal metabolic function with no sign of other autoimmune conditions.
Reports on correlations between RESLES and neuronal or glial antibodies are extremely rare, only previously described in only one anti-NMDAR encephalitis, one anti-VGKC encephalitis, and one anti-Yo encephalitis case, respectively [3-5]. Recently, autoimmune GFAP astrocytopathy in an adult with viral infection and a child with hyponatremia was reported, showing SCC lesions [7, 8]. However, viral infections and hyponatremia itself can also cause RESLES, consequently not clarifying the role of anti-GFAP antibodies in RESLES. Therefore, with exclusion of other etiologies, this is the first case revealing direct links between anti-GFAP antibodies and RESLES.

Possible mechanism of RESLES in autoimmune GFAP astrocytopathy could be inflammatory infiltrations. Pathological studies have showed extensive inflammation, particularly around small vessels in all autoimmune GFAP astrocytopathy cases [6]. The rich vascular supply of the 
splenium mainly by the vertebrobasilar system could have led to intense inflammatory infiltrations in this area, leading to splenial lesion [4].

In most cases, encephalitis/encephalopathy with reversible splenial lesion showed relatively mild CNS manifestation and completely recovered within 1 month, sometimes also termed as mild encephalitis/encephalopathy with a reversible splenial lesion (MERS) [9]. However, recent studies have indicated that encephalitis/encephalopathy with reversible splenial lesion could not be simply considered as a mild clinical disorder, especially in patients who required ventilator supporting in acute phase of the disease [10]. Although there had been no established treatment guidelines, immunotherapy seems to be effective for neuronal/glial antibodyrelated RESLES based on limited evidences [3-5].

In conclusion, anti-GFAP autoimmunity should be added to the list of potential causes of RESLES. Autoimmune GFAP astrocytopathy should be considered when brain MRI confirms RESLES. Timely immunotherapy including corticosteroid and IVIg may improve the symptoms and benefit the long-term outcomes. Further study of future cases can promote a better understanding of the specific mechanism of autoimmune neurological antibody associated RESLES.

Acknowledgements The authors thank the study participant for the participation in our study.

Author contribution K. Guo and X. Lai collected data and drafted the manuscript. Y. Liu revised the figure. D. Zhou revised the manuscript. Z. Hong conceptualized and revised the manuscript.

Funding Supported by the National Natural Science Foundation of China (grants 81671291 and 81971213).

Data statement Further anonymized data can be made available to qualified investigators upon request to the corresponding author.

\section{Declarations}

Ethical approval None.

Conflict of interest The authors declare no competing interests.

\section{References}

1. Garcia-Monco JC, Cortina IE, Ferreira E et al (2011) Reversible splenial lesion syndrome (RESLES): what's in a name? J Neuroimaging 21(2):e1-14. https://doi.org/10.1111/j.1552-6569.2008. 00279.x

2. Liu J, Liu D, Yang B et al (2017) Reversible splenial lesion syndrome (RESLES) coinciding with cerebral venous thrombosis: a report of two cases. Ther Adv Neurol Disord 10(12):375-379. https://doi.org/10.1177/1756285617727978

3. Kaminski JA, Pruss H (2019) N-methyl-d-aspartate receptor encephalitis with a reversible splenial lesion. Eur J Neurol 26(6):e68-e69. https://doi.org/10.1111/ene.13900

4. Gilder TR, Hawley JS, Theeler BJ (2016) Association of reversible splenial lesion syndrome (RESLES) with Anti-VGKC autoantibody syndrome: a case report. Neurol Sci 37(5):817-819. https:// doi.org/10.1007/s10072-015-2464-y

5. Renard D, Taieb G, Briere C et al (2012) Mild encephalitis/ encephalopathy with a reversible splenial, white matter, putaminal, and thalamic lesions following anti-Yo rhombencephalitis. Acta Neurol Belg 112(4):405-407. https://doi.org/10.1007/ s13760-012-0080-7

6. Shan F, Long Y, Qiu W (2018) Autoimmune glial fibrillary acidic protein astrocytopathy: a review of the literature. Front Immunol 9:2802. https://doi.org/10.3389/fimmu.2018.02802

7. Oger V, Bost C, Salah L et al (2020) Mild encephalitis/encephalopathy with reversible splenial lesion syndrome: an unusual presentation of anti-GFAP astrocytopathy. Eur J Paediatr Neurol 26:89-91. https://doi.org/10.1016/j.ejpn.2020.03.002

8. Issa N, Martin C, Dulau C et al (2020) Severe anti-GFAP meningo-encephalomyelitis following viral infection. Mult Scler Relat Disord 45:102448. https://doi.org/10.1016/j.msard.2020. 102448

9. Tada H, Takanashi J, Barkovich AJ et al (2004) Clinically mild encephalitis/encephalopathy with a reversible splenial lesion. Neurology 63(10):1854-1858. https://doi.org/10.1212/01.wnl. 0000144274.12174.cb

10. Zhu Y, Zheng J, Zhang L et al (2016) Reversible splenial lesion syndrome associated with encephalitis/encephalopathy presenting with great clinical heterogeneity. BMC Neurol 16:49. https://doi. org/10.1186/s12883-016-0572-9

Publisher's note Springer Nature remains neutral with regard to jurisdictional claims in published maps and institutional affiliations. 